\title{
Addition and correction
}

\section{A Versatile Enantioselective Synthesis of Barrenazines}

\author{
Miguel Peña-López, M. Montserrat Martínez, Luis A. Sarandeses, ${ }^{*}$ and José Pérez Sestelo* \\ Departamento de Química Fundamental, Universidade da Coruña, E-15071 A Coruña, Spain \\ sestelo@udc.es; qfsarand@udc.es
}

\section{Supporting Information}

The stereochemistry of epoxide $\mathbf{1 0}$ was assigned through chemical derivatization and X-ray analysis of compound 19, prepared following the synthetic sequence shown in the scheme.

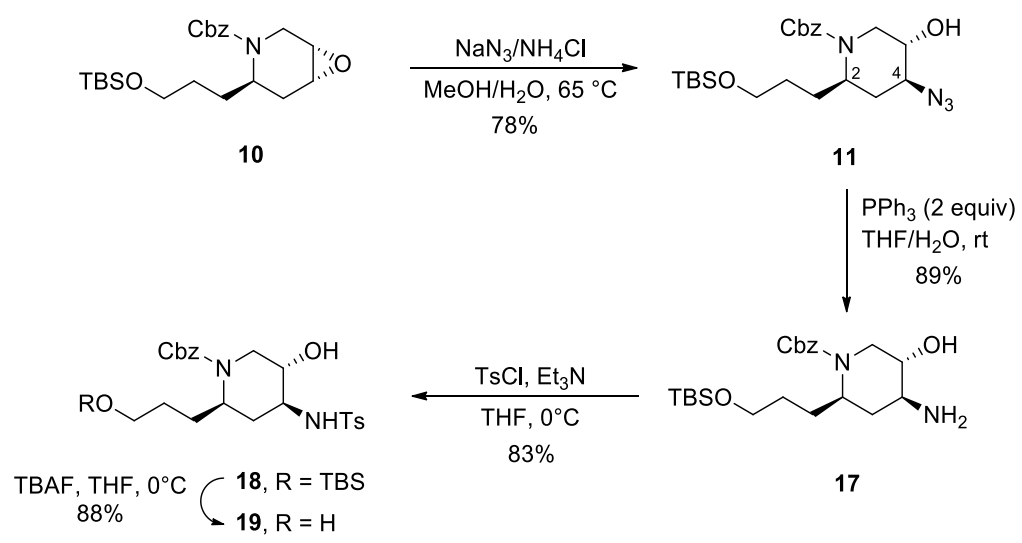

\section{Experimental}

$(2 R, 4 S, 5 S)$-Benzyl

4-amino-2-(3-((tert-butyldimethylsilyl)oxy)propyl)-5hydroxypiperidine-1-carboxylate (17). To a mixture of azido alcohol 11 (303.5 mg, 0.676 mmol) in THF: $\mathrm{H}_{2} \mathrm{O}(8 \mathrm{~mL}, 3: 1)$ at $\mathrm{rt}, \mathrm{Ph}_{3} \mathrm{P}(372.5 \mathrm{mg}, 1.42 \mathrm{mmol})$ was added and the reaction mixture stirred overnight. The solvent was evaporated at reduced pressure and the residue purified by column chromatography $\left(20 \% \mathrm{MeOH} / \mathrm{CH}_{2} \mathrm{Cl}_{2}\right)$ to give amino alcohol $17(254 \mathrm{mg}$, $0.601 \mathrm{mmol}, 89 \%)$ as a yellow oil. ${ }^{1} \mathrm{H} \mathrm{NMR}\left(300 \mathrm{MHz}, \mathrm{CDCl}_{3}, 25^{\circ} \mathrm{C}\right): \delta 0.01(\mathrm{~s}, 6 \mathrm{H}), 0.87(\mathrm{~s}$, $9 \mathrm{H}), 1.19-1.31(\mathrm{~m}, 2 \mathrm{H}), 1.43-1.57(\mathrm{~m}, 2 \mathrm{H}), 1.76-1.86(\mathrm{~m}, 1 \mathrm{H}), 1.95-2.04(\mathrm{~m}, 1 \mathrm{H}), 2.65$ (br s, 
$3 \mathrm{H}), 2.84-2.90(\mathrm{~m}, 1 \mathrm{H}), 3.41(\mathrm{dd}, J=3.8,14.4 \mathrm{~Hz}, 1 \mathrm{H}), 3.50-3.61(\mathrm{~m}, 3 \mathrm{H}), 3.82(\mathrm{~d}, J=14.4$ $\mathrm{Hz}, 1 \mathrm{H}), 4.01(\mathrm{q}, J=6.9 \mathrm{~Hz}, 1 \mathrm{H}), 5.06-5.17(\mathrm{~m}, 2 \mathrm{H}), 7.28-7.36(\mathrm{~m}, 5 \mathrm{H}) \mathrm{ppm} ;{ }^{13} \mathrm{C}$ NMR $(75$ $\left.\mathrm{MHz}, \mathrm{CDCl}_{3}, 25^{\circ} \mathrm{C}\right): \delta-5.3\left(2 \times \mathrm{CH}_{3}\right), 18.3(\mathrm{C}), 25.9\left(3 \times \mathrm{CH}_{3}\right), 29.6\left(\mathrm{CH}_{2}\right), 29.7\left(\mathrm{CH}_{2}\right)$, $33.3\left(\mathrm{CH}_{2}\right), 44.4\left(\mathrm{CH}_{2}\right), 52.4(2 \times \mathrm{CH}), 62.8\left(\mathrm{CH}_{2}\right), 67.1\left(\mathrm{CH}_{2}\right), 72.9(\mathrm{CH}), 127.8(2 \times \mathrm{CH})$, $127.9(\mathrm{CH}), 128.5$ (2 x CH), 136.7 (C), 156.5 (C) ppm; IR (ATR): 3033, 2951, 2927, 2855, 2359, 2341, 1691, $1498 \mathrm{~cm}^{-1}$; MS (ESITOF) $\mathrm{m} / z(\%): 423\left(M^{+}+\mathrm{H}, 51\right), 379$ (2); HRMS (ESITOF) $m / z$ calcd for $\mathrm{C}_{22} \mathrm{H}_{39} \mathrm{O}_{4} \mathrm{~N}_{2} \mathrm{Si}\left(M^{+}+\mathrm{H}\right), 423.2673$; found, 423.2679 .

$(2 R, 4 S, 5 S)$-Benzyl

2-(3-((tert-butyldimethylsilyl)oxy)propyl)-5-hydroxy-4-(4methylphenylsulfonamido)piperidine-1-carboxylate (18). To a solution of amino alcohol 17 (44 mg, $0.104 \mathrm{mmol})$ and $\mathrm{Et}_{3} \mathrm{~N}(73 \mu \mathrm{L}, 0.520 \mathrm{mmol})$ in $\mathrm{CH}_{2} \mathrm{Cl}_{2}(5 \mathrm{~mL})$ at $0{ }^{\circ} \mathrm{C}$, a solution of tosyl chloride $(60.0 \mathrm{mg}, 0.312 \mathrm{mmol})$ in $\mathrm{CH}_{2} \mathrm{Cl}_{2}(3 \mathrm{~mL})$ was added via cannula, and the mixture was warmed at $\mathrm{rt}$ overnight. The reaction was quenched by addition of water $(5 \mathrm{~mL})$ and extracted with EtOAc $(3 \times 10 \mathrm{~mL})$, washed with brine $(20 \mathrm{~mL})$, dried with $\mathrm{MgSO}_{4}$, filtered and the solvent removed under reduced pressure. Purification by column chromatography (20\% $\left.\mathrm{MeOH} / \mathrm{CH}_{2} \mathrm{Cl}_{2}\right)$ afforded $18(48 \mathrm{mg}, 0.083 \mathrm{mmol}, 83 \%)$ as a heavy oil. ${ }^{1} \mathrm{H}$ NMR $\left(300 \mathrm{MHz}, \mathrm{CDCl}_{3}, 25^{\circ} \mathrm{C}\right): \delta 0.02(\mathrm{~s}, 6 \mathrm{H}), 0.88(\mathrm{~s}, 9 \mathrm{H}), 1.22-1.34(\mathrm{~m}, 2 \mathrm{H}), 1.38-1.54$ $(\mathrm{m}, 2 \mathrm{H}), 1.65-1.76(\mathrm{~m}, 1 \mathrm{H}), 1.93-2.05(\mathrm{~m}, 1 \mathrm{H}), 2.44(\mathrm{~s}, 3 \mathrm{H}), 2.71(\mathrm{~d}, J=3.6 \mathrm{~Hz}, 1 \mathrm{H}), 3.07-$ $3.15(\mathrm{~m}, 1 \mathrm{H}), 3.29(\mathrm{dd}, J=3.4,14.8 \mathrm{~Hz}, 1 \mathrm{H}), 3.49-3.60(\mathrm{~m}, 2 \mathrm{H}), 3.76$ (br s, $1 \mathrm{H}), 3.92$ (d, $J=$ $14.8 \mathrm{~Hz}, 1 \mathrm{H}), 3.85-4.04(\mathrm{~m}, 1 \mathrm{H}), 4.97$ (d, $J=6.1 \mathrm{~Hz}, 1 \mathrm{H}), 5.06-5.16(\mathrm{~m}, 2 \mathrm{H}), 7.28-7.39$ (m, $7 \mathrm{H}), 7.76(\mathrm{~d}, J=8.3 \mathrm{~Hz}, 2 \mathrm{H}) \mathrm{ppm} ;{ }^{13} \mathrm{C} \mathrm{NMR}\left(75 \mathrm{MHz}, \mathrm{CDCl}_{3}, 25{ }^{\circ} \mathrm{C}\right): \delta-5.3\left(2 \times \mathrm{CH}_{3}\right), 18.3$ (C), $21.5\left(\mathrm{CH}_{3}\right), 25.9\left(3 \times \mathrm{CH}_{3}\right), 29.3\left(\mathrm{CH}_{2}\right), 29.7\left(\mathrm{CH}_{2}\right), 30.9\left(\mathrm{CH}_{2}\right), 43.4\left(\mathrm{CH}_{2}\right), 51.6(\mathrm{CH})$, 54.6 (CH), $62.6\left(\mathrm{CH}_{2}\right), 67.3\left(\mathrm{CH}_{2}\right), 70.9(\mathrm{CH}), 127.1(2 \times \mathrm{CH}), 127.8(2 \times \mathrm{CH}), 128.0(\mathrm{CH})$, 128.5 (2 x CH), 129.9 (2 x CH), 136.5 (C), 136.6 (C), 143.9 (C), 156.3 (C) ppm; IR (ATR): 3466, 3261, 2952, 2927, 2856, $1671 \mathrm{~cm}^{-1}$; MS (ESITOF) $m / z(\%): 577\left(M^{+}+\mathrm{H}, 78\right), 533(8)$; HRMS (ESITOF) $m / z$ calcd for $\mathrm{C}_{29} \mathrm{H}_{45} \mathrm{O}_{6} \mathrm{~N}_{2} \mathrm{SSi}\left(M^{+}+\mathrm{H}\right), 577.2762$; found, 577.2753.

$(2 R, 4 S, 5 S)$-Benzyl

5-hydroxy-2-(3-hydroxypropyl)-4-(4-

methylphenylsulfonamido)piperidine-1-carboxylate (19). To a cold solution of 18 (48 $\mathrm{mg}$, $0.083 \mathrm{mmol})$ in THF $(3 \mathrm{~mL})$ at $0{ }^{\circ} \mathrm{C}$, a solution of TBAF $(420 \mu \mathrm{L}, 0.416 \mathrm{mmol}, 1 \mathrm{M}$ in THF $)$ was added and the resulting mixture stirred overnight. The solvent was evaporated at reduced pressure and the residue purified by column chromatography $\left(20 \% \mathrm{MeOH} / \mathrm{CH}_{2} \mathrm{Cl}_{2}\right)$ to give 19 (34 mg, $0.073 \mathrm{mmol}, 88 \%$ ) as a white solid (mp 137-139 $\left.{ }^{\circ} \mathrm{C}, \mathrm{CH}_{2} \mathrm{Cl}_{2}\right) .{ }^{1} \mathrm{H} \mathrm{NMR}(300 \mathrm{MHz}$, $\left.\mathrm{CDCl}_{3}, 25^{\circ} \mathrm{C}\right): \delta 1.36-1.85(\mathrm{~m}, 5 \mathrm{H}), 1.96-2.05(\mathrm{~m}, 1 \mathrm{H}), 2.43(\mathrm{~s}, 3 \mathrm{H}), 3.11(\mathrm{q}, J=5.5 \mathrm{~Hz}, 1 \mathrm{H})$, $3.36(\mathrm{dd}, J=2.9,14.7 \mathrm{~Hz}, 1 \mathrm{H}), 3.53-3.69(\mathrm{~m}, 2 \mathrm{H}), 3.80-3.84(\mathrm{~m}, 1 \mathrm{H}), 3.92$ (d, $J=14.7 \mathrm{~Hz}$, $1 \mathrm{H}), 4.05(\mathrm{q}, J=6.5 \mathrm{~Hz}, 1 \mathrm{H}), 5.07-5.17(\mathrm{~m}, 2 \mathrm{H}), 5.65(\mathrm{~d}, J=5.5 \mathrm{~Hz}, 1 \mathrm{H}), 7.30-7.37(\mathrm{~m}, 7 \mathrm{H})$, $7.76(\mathrm{~d}, J=8.3 \mathrm{~Hz}, 2 \mathrm{H}) \mathrm{ppm} ;{ }^{13} \mathrm{C} \mathrm{NMR}\left(75 \mathrm{MHz}, \mathrm{CDCl}_{3}, 25{ }^{\circ} \mathrm{C}\right): \delta 21.5\left(\mathrm{CH}_{3}\right), 28.6\left(\mathrm{CH}_{2}\right)$, $29.2\left(\mathrm{CH}_{2}\right), 29.3\left(\mathrm{CH}_{2}\right), 42.6\left(\mathrm{CH}_{2}\right), 51.0(\mathrm{CH}), 53.6(\mathrm{CH}), 62.3\left(\mathrm{CH}_{2}\right), 67.3\left(\mathrm{CH}_{2}\right), 69.8(\mathrm{CH})$, $127.2(2 \times \mathrm{CH}), 127.8(2 \times \mathrm{CH}), 128.2(\mathrm{CH}), 128.5(2 \times \mathrm{CH}), 129.9(2 \times \mathrm{CH}), 136.4(\mathrm{C})$, 
136.5 (C), 143.9 (C), 156.3 (C) ppm; IR (ATR): 3424, 2936, 2921, 2358, 2325, $1670 \mathrm{~cm}^{-1}$; MS (ESITOF) $m / z$ (\%): $463\left(M^{+}+\mathrm{H}, 31\right), 419$ (100); HRMS (ESITOF) $\mathrm{m} / z$ calcd for $\mathrm{C}_{23} \mathrm{H}_{31} \mathrm{O}_{6} \mathrm{~N}_{2} \mathrm{~S}\left(M^{+}+\mathrm{H}\right), 463.1897$; found, 463.1890 . 

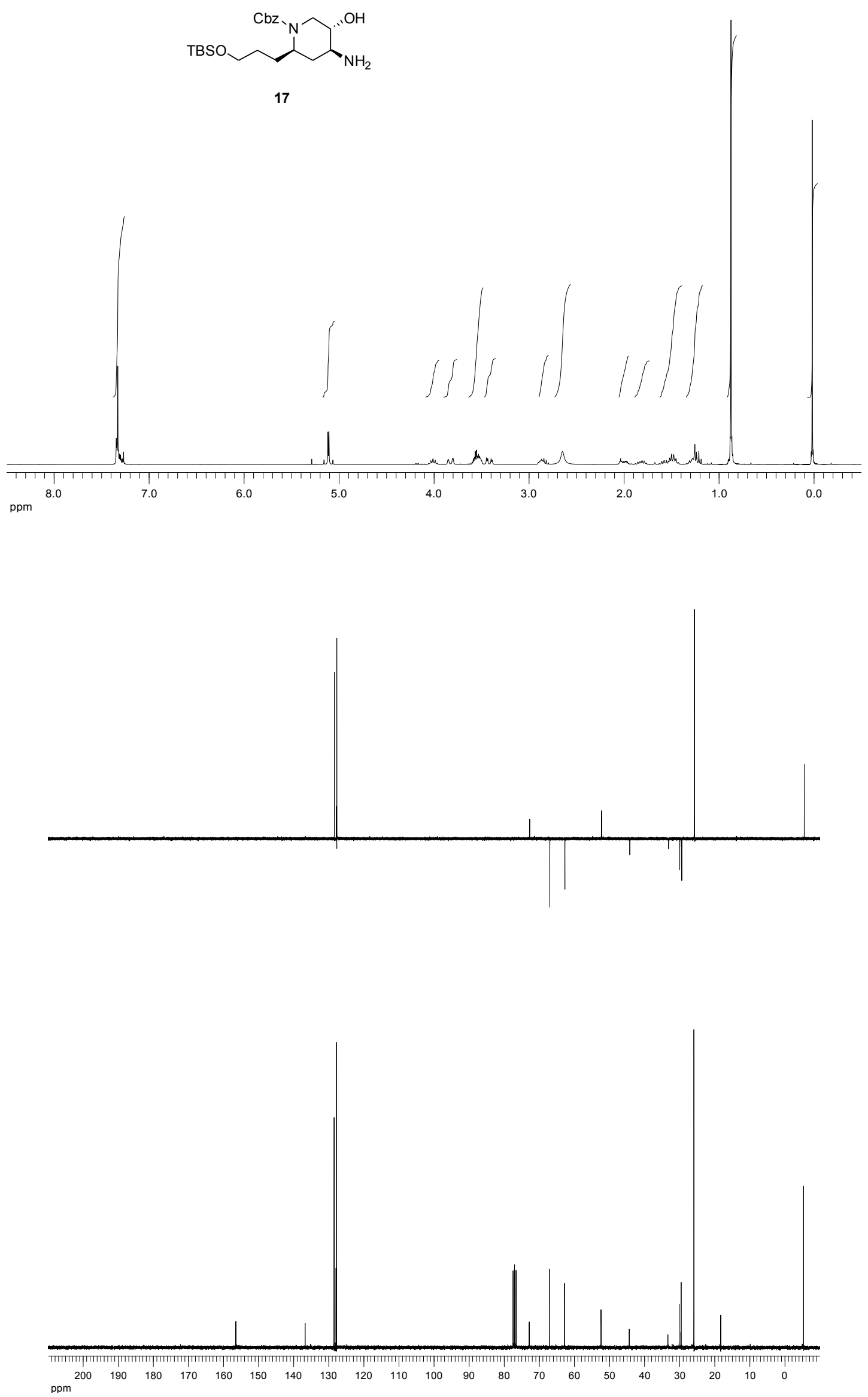


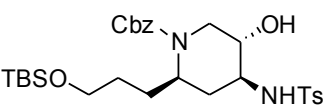

18

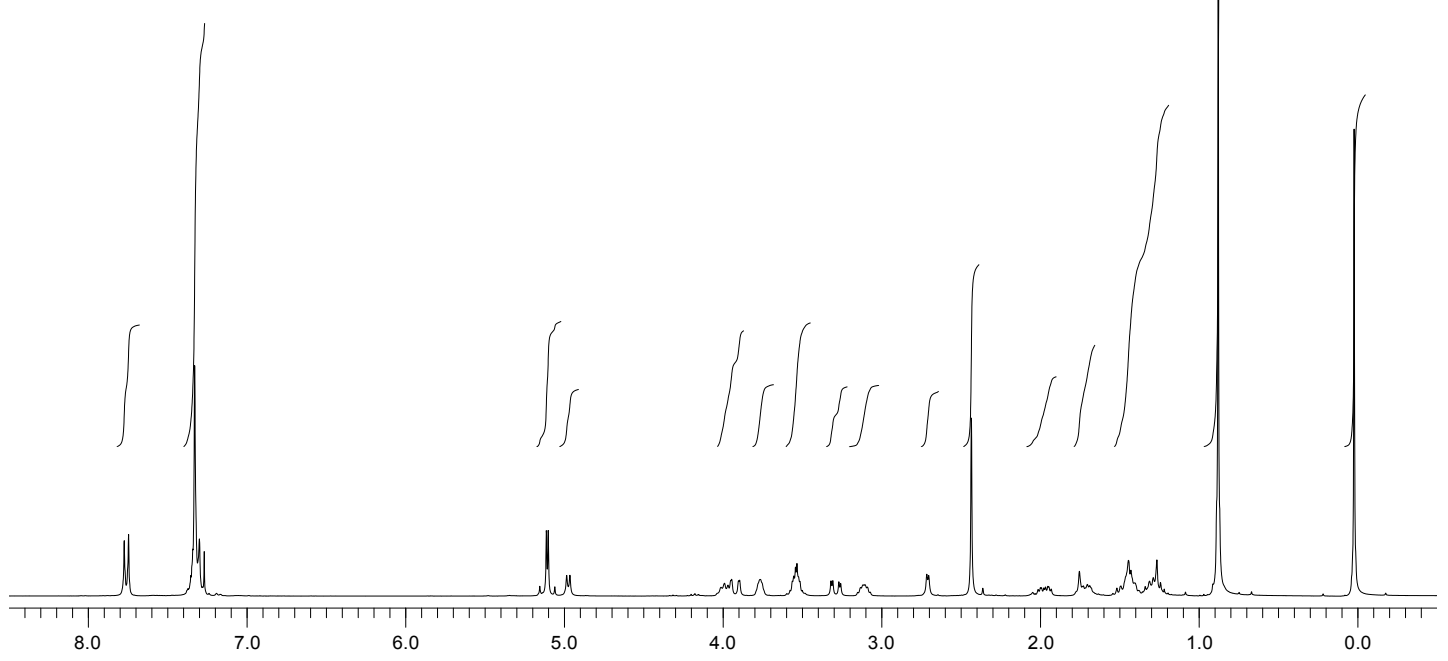

ppm
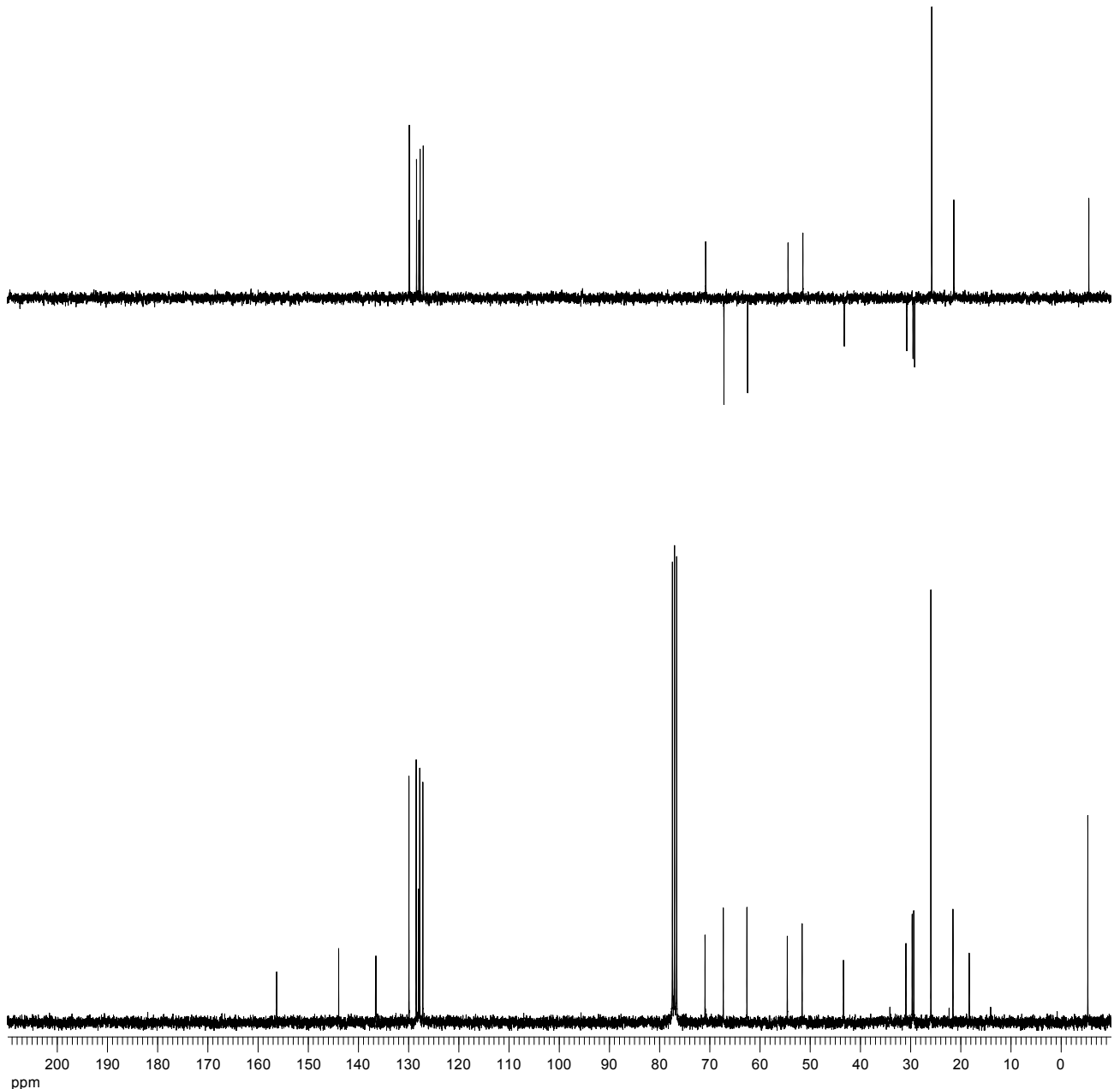

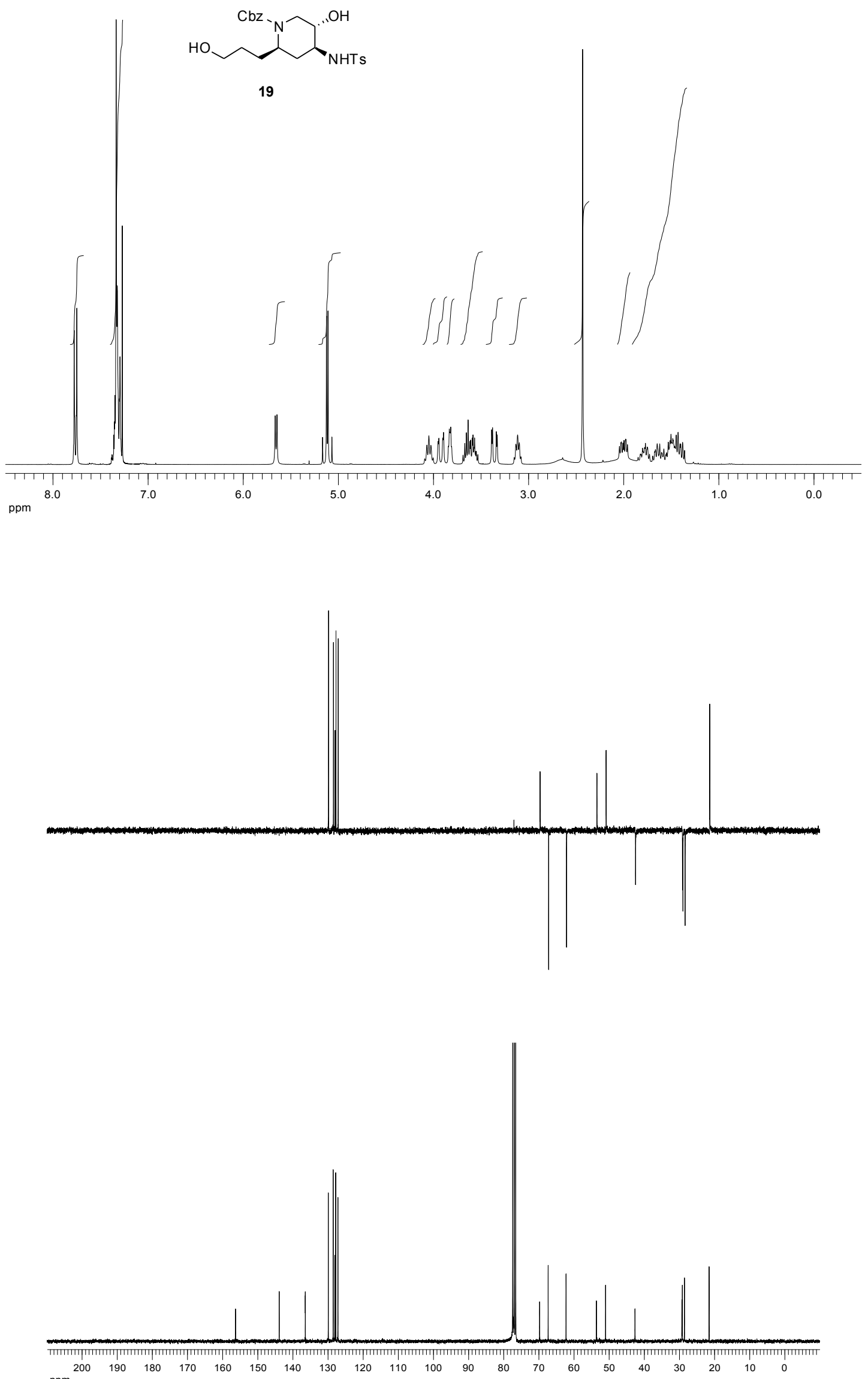